\title{
DNA copy number alterations in pleomorphic leiomyosarcoma: A case report
}

\author{
MASAHIKO KANAMORI ${ }^{1,2}$, TAKETOSHI YASUDA ${ }^{2}$, SHIGEHARU NOGAMI $^{2}$, \\ KAYO SUZUKI $^{2}$ and TAKESHI HORI ${ }^{2}$ \\ Departments of ${ }^{1}$ Human Science and ${ }^{2}$ Orthopaedics, University of Toyama, Toyama City, Toyama 930-0194, Japan
}

Received July 19, 2013; Accepted January 24, 2014

DOI: $10.3892 / \mathrm{ol} .2014 .2030$

\begin{abstract}
Pleomorphic leiomyosarcoma (P-LMS) is a rare morphological variant of LMS. The current study presents the cytogenetic data of a P-LMS that arose in the axillary region of a 31-year-old male. The results of array-based comparative genomic hybridization for the primary tumor showed DNA copy number alteration (DCNA) gains of 8ptel, 17ptel and $17 \mathrm{q} 11.2$ and losses of 2ptel, 7ptel, 7qtel, 10p15, 12p12-13.1, 13q14.2-14.3, 15q25-26 and Yq11. However, a metastatic lesion showed cytogenetic data different from the primary tumor DCNAs, with only the locus of 17ptel (282M15/SP6) in common between them. These observations add to the spectrum of DCNAs that have been reported in previous cases of LMS and provide novel cytogenetic data.
\end{abstract}

\section{Introduction}

Previous cytogenetic studies have revealed that complex genetic changes occur in leiomyosarcoma (LMS). Numerical aberrations and structural rearrangements in all chromosomes have been reported in LMS $(1,2)$. No specific primary cytogenetic abnormality characteristics of LMS have been identified, although, consistent chromosomal profiles have been observed and an attempt at a chromosomal classification has been made (1). Recent advances in mapping resolution using array-based comparative genomic hybridization (array $\mathrm{CGH}$ ) have significantly improved the resolving power in comparison with that of metaphase $\mathrm{CGH}(3,4)$. This has provided more information regarding the complexity and exact locations of genomic rearrangements, which result in DNA copy number alterations (DCNAs). Using array $\mathrm{CGH}$, it has previously been reported that an increase in DCNAs is associated with tumor size in LMS (5).

Correspondence to: Professor Masahiko Kanamori, Department of Human Science, University of Toyama, 2630 Sugitani, Toyama City, Toyama 930-0194, Japan

E-mail: kanamori@med.u-toyama.ac.jp

Key words: leiomyosarcoma, comparative genomic hybridization, DNA, chromosome
As a morphological variant of LMS, pleomorphic LMS (P-LMS) was initially described as an important differential diagnosis of pleomorphic malignant fibrous histiocytoma by Fletcher in 1992 (6). Subsequently, various studies have examined a series of P-LMS using morphological, immunohistochemical and electron microscopy methods, establishing the occurrence of this variant (7-9). However, cytogenetic and molecular genetic data for P-LMS have not previously been described. Therefore, the current study reports the DCNA observations for a primary tumor and metastatic lymph node in a patient presenting with P-LMS.

\section{Case report}

A 31-year-old male presented with a mass in the left axilla, which had rapidly enlarged over the previous two months prior to admission to the University Hospital of Toyama (Toyoma, Japan). The patient had an unremarkable clinical history. On examination, a 10x9 $\mathrm{cm}$ elastic hard mass, presenting with tenderness, was identified. Neurovascular examination results and laboratory observations were relatively normal. T1- and T2-weighted magnetic resonance imaging showed a large cystic tumor with fluid-fluid levels, indicative of a soft tissue sarcoma (Fig. 1). Angiograms revealed that the tumor was hypervascular.

An open tumor biopsy was performed and the pathology was determined as a P-LMS (Fig. 2). Following preoperative chemotherapy for two days with doxorubicin (40 mg/day) and ifosfamide ( $4 \mathrm{~g} / \mathrm{day}$ ), the tumor was surgically resected. Gross examination of the resected specimen showed an elastic firm and tan-to-yellow mass; its largest diameter was $14 \mathrm{~cm}$. The tumor had a large cyst that contained a brown serous fluid. An enlarged regional lymph node (metastasis) was also resected.

Microscopically, the tumor consisted of myxomatous and pleomorphic areas with proliferating ovoid or short-spindle atypical cells and a number of mitotic figures were observed (Fig. 2). Immunohistochemistry revealed that the tumor cells were positive for vimentin, h-caldesmon and muscle actin (HHF-35; Fig. 3), with slight positivity for smooth muscle actin (SMA). However, these cells were negative for desmin, S-100, cluster of differentiation (CD)31, CD34, CD56, CD99, cytokeratin, c-kit, calretinin and p53 (DakoCytomation, Copenhagen, Denmark). 

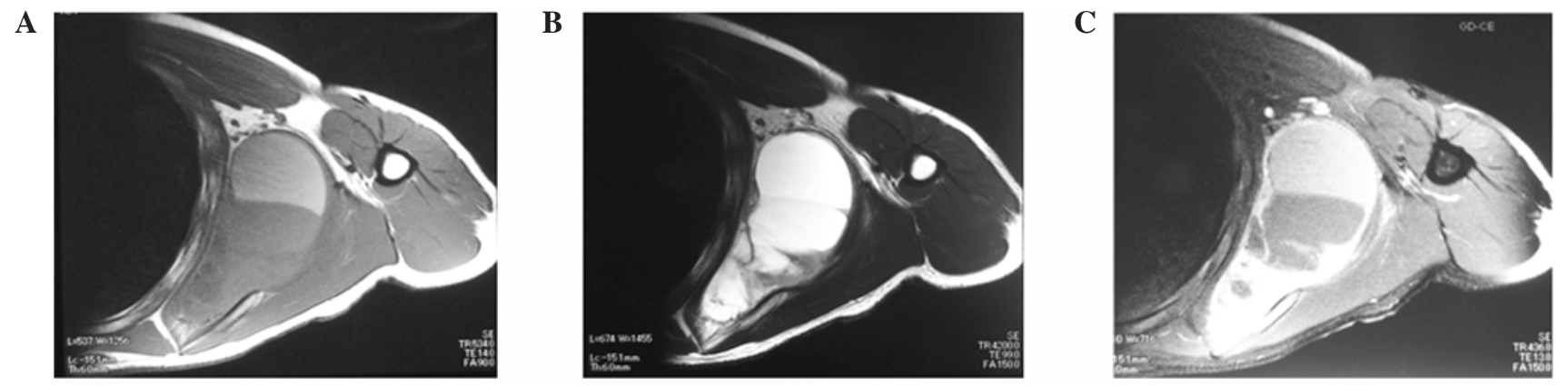

Figure 1. (A) Axial T1-weighted (TR/TE, 534/14) spin echo image of the left axilla showed a cystic tumor with fluid-fluid level, indicative of a soft tissue sarcoma. (B) Axial T2-weighted (TR/TE, 4200/99) spin echo image showed a large cystic tumor. (C) Gadopentetic acid heterogeneously enhanced the mass (TR/TE, 436/13). TR/TE; repetition time/echo time.
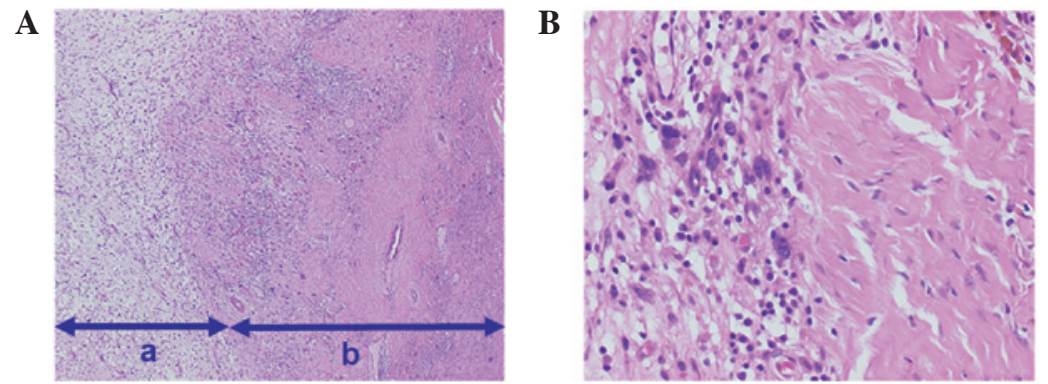

Figure 2. (A) The tumor was composed of (a) myxomatous and (b) pleomorphic areas (H\&E; magnification, x40). (B) The pleomorphic area contained proliferative ovoid or short-spindle atypical cells and a number of mitotic figures were present (H\&E; magnification, x100).

A

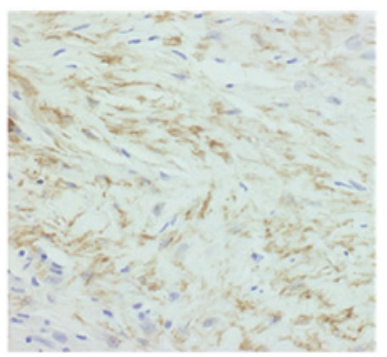

B

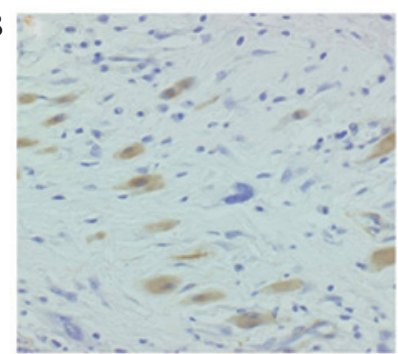

C

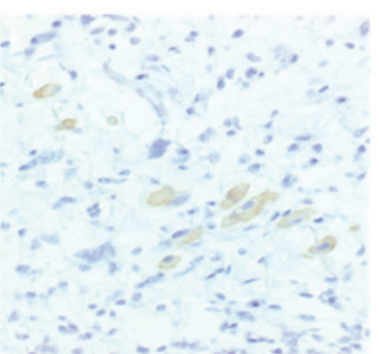

Figure 3. Tumor cells were immunoreactive to (A) smooth muscle actin, (B) h-caldesmon and (C) muscle actin (HHF-35) (immunohistochemical staining; magnification, $\mathrm{x} 100$ )

A

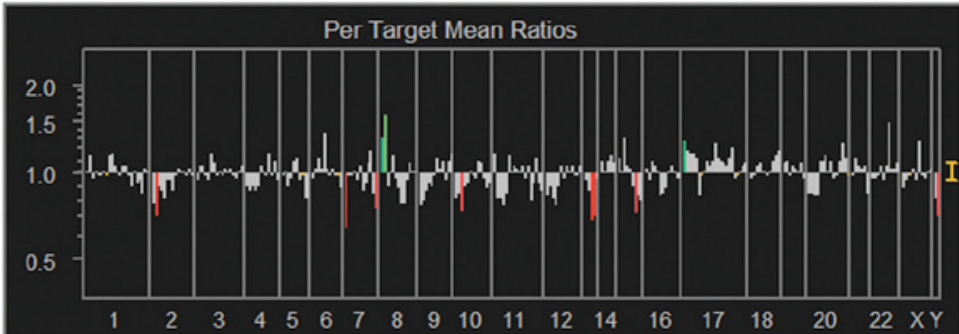

B

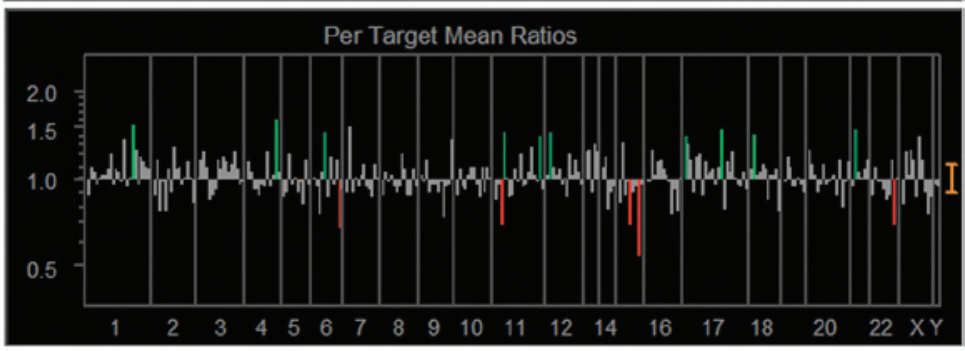

Figure 4. Compared with the primary lesion (A), the genetic aberrations in the metastatic lesion (B) had increased. 
Table I. Summary of DCNA data in the current case of pleomorphic LMS.

A, Gain

\begin{tabular}{|c|c|c|c|c|c|}
\hline \multicolumn{3}{|c|}{ Primary tumor } & \multicolumn{3}{|c|}{ Lymph node metastasis } \\
\hline Gene name & Locus & DCNAs & Gene name & Locus & DCNAs \\
\hline D8S504 & $8 \mathrm{p}$ tel & 1.31 & WI-5663,WI-13414 & $1 q 21$ & 1.54 \\
\hline D8S596 & $8 p$ tel & 1.56 & 4QTEL11 & $4 \mathrm{q}$ tel & 1.59 \\
\hline $282 M 15 / S P 6$ & $17 \mathrm{p}$ tel & 1.29 & HTRlB & $6 q 13$ & 1.44 \\
\hline \multirow[t]{8}{*}{$N F 13^{\prime}$} & $17 q 11.2$ & 1.26 & $C D K N 1 C(p 57)$ & $11 \mathrm{p} 15.5$ & 1.45 \\
\hline & & & WI-6509 & $11 q$ tel & 1.39 \\
\hline & & & SHGC-5557 & $12 \mathrm{p}$ tel & 1.44 \\
\hline & & & $S N R P N$ & $15 q 12$ & 1.34 \\
\hline & & & $282 M 15 / S P 6$ & $17 \mathrm{p}$ tel & 1.39 \\
\hline & & & $\operatorname{PPARBP}(P B P)$ & $17 q 12$ & 1.48 \\
\hline & & & SHGC17327 & $18 \mathrm{p}$ tel & 1.42 \\
\hline & & & RUNX1(AML1) & $21 \mathrm{q} 22.3$ & 1.49 \\
\hline
\end{tabular}

B, Loss

\begin{tabular}{|c|c|c|c|c|c|}
\hline \multicolumn{3}{|c|}{ Primary tumor } & \multicolumn{3}{|c|}{ Lymph node metastasis } \\
\hline Gene name & Locus & DCNAs & Gene name & Locus & DCNAs \\
\hline 2PTEL27 & $2 \mathrm{p}$ tel & 0.72 & ESRl & $6 q 25.1$ & 0.69 \\
\hline G31341 & $7 \mathrm{p}$ tel & 0.65 & $H R A S$ & $11 \mathrm{p} 15.5$ & 0.71 \\
\hline 7QTEL2O & $7 \mathrm{q}$ tel & 0.76 & $M A P 2 K 5$ & $15 q 23$ & 0.70 \\
\hline D10S249,D10S533 & $10 \mathrm{p} 15$ & 0.75 & PACE4C & $15 q$ tel & 0.55 \\
\hline$C D K N 1 B(p 27)$ & 12p13.1-p12 & 0.78 & stSG30213 & $16 \mathrm{q}$ tel & 0.78 \\
\hline$D 13 S 319$ & $13 q 14.2$ & 0.69 & $A R S A$ & $22 \mathrm{q}$ tel & 0.71 \\
\hline$D 13 S 25$ & $13 q 14.3$ & 0.72 & & & \\
\hline$I G F 1 R$ & $15 q 25-q 26$ & 0.74 & & & \\
\hline AZFa region & Yq11 & 0.72 & & & \\
\hline
\end{tabular}

DCNAs, DNA copy number alterations; LMS, leiomyosarcoma.

The tumor specimens were frozen and stored at $-80^{\circ} \mathrm{C}$ until the $\mathrm{CGH}$ analysis was conducted. Genomic DNA was isolated from a tumor sample by standard procedures using proteinase $\mathrm{K}$ digestion and phenol-chloroform extraction $(10,11)$. Hybridization and analysis by array CGH were performed according to the manufacturer's instructions (Vysis-Abbott Japan, Inc., Tokyo, Japan). The array CGH comprised of 287 clones that included important tumor suppressors and oncogene loci. Tumor DNA $(n=100)$ was labeled by random priming with fluorescent-labeled cy3-dUTP (Perkin-Elmer, Waltham, MA, USA) and normal reference DNA ( $=100)$ was labeled with cy5-dUTP. The tumor and control DNAs were mixed with Cot-1 DNA (Vysis-Abbott Japan, Inc.), precipitated and resuspended in a microarray hybridization buffer, which contained $50 \%$ formamide. The hybridization solution was heated to $80^{\circ} \mathrm{C}$ for $10 \mathrm{~min}$ to denature the DNA and subsequently incubated for $1 \mathrm{~h}$ at $37^{\circ} \mathrm{C}$. Hybridization was performed for $72 \mathrm{~h}$ in a moist chamber, followed by post-hybridization washing in a $50 \%$ formamide/ $2 \mathrm{X}$ saline sodium citrate buffer at $45^{\circ} \mathrm{C}$. Slides were mounted in phosphate-buffered saline containing 4',6-diamidino-2-phenylindole (array DAPI solution). Fluorescence intensity images were captured from the hybridized microarray slides using a GenoSensor Reader System equipped with Array 300 Software (Vysis-Abbott Japan, Inc.). The total intensity and intensity ratio of the two dyes for each spot were automatically calculated. The diagnostic cut-off levels representing the gains and losses of the DCNAs were set to an upper threshold of 1.25 and a lower threshold of 0.75 .

The current study was approved by the Institutional Review Board for Human Use of the University Hospital of Toyama. Written informed consent for the publication of the patient's data was obtained from the patient.

The genetic instabilities of the primary tumor and metastatic lymph node were analyzed and compared in the present study. The DCNAs of all chromosomes were determined (Fig. 4). DCNAs that exhibited marked gains (1.25) or losses $(0.75)$ were selected and are listed in Table I. Of 
the 287 clones represented on array CGH, the primary tumor showed four DCNAs (1.4\%) with gains and nine DCNAs (3.1\%) with losses. In comparison, the metastatic lymph node showed 11 DCNAs (3.8\%) with gains and six DCNAs $(2.1 \%)$ with losses. Genetic aberrations in the metastatic lesion were increased compared with the primary lesion. Only one DCNA, 17ptel (282M15/SP6), was increased in the two samples.

The current study focused on the gain of 8ptel (D8S504 and D8S596) and the loss of 13q14.2-14.3 (D13S319 and D13S25) in the primary tumor, since these loci showed marked changes on the two different arrays for the same locus. These DCNAs may provide various starting points for identifying candidate genes that are associated with oncogenesis. However, these genetic changes were not observed in the metastatic lymph node (Table I).

\section{Discussion}

Analysis of DCNAs to identify the molecular events in soft tissue sarcomas is important. Array CGH technology $(3,4)$ enables the detection of specific genes with DCNAs and may be used to screen for genomic imbalances in human solid cancers. The key biological value of high-resolution array $\mathrm{CGH}$ is its ability to detect small amplicons and deletions that potentially harbor specific oncogenes or suppressor genes.

P-LMS is usually defined by an association between areas of undifferentiated pleomorphic sarcoma and areas showing morphological, immunohistochemical or ultrastructural evidence of smooth muscle differentiation. SMA, desmin and HHF-35 are specific to smooth muscle. The novel marker, h-caldesmon, is highly specific for smooth muscle differentiation, however, is expressed in only $40 \%$ of cases (9). To date, $\mathrm{CGH}$ analysis has not been performed to differentiate LMS subtypes. Therefore, we were unable discuss P-LMS DCNAs from previous reports.

Riva et al (12) previously identified a $19 \mathrm{p}$ deletion in a single case of recurring LMS. In addition, a previous CGH analysis of 28 cases of LMS (13) reported that a 13q14-q21 loss and 5p14-pter gain at diagnosis may be used to identify patients with LMS who are likely to have reduced survival times. The most frequent losses detected were 10q (20 cases; 69\%) and 13q (17 cases; 59\%), the most frequent gain that was detected was $17 \mathrm{p}$ (16 cases; 55\%), and the high-level amplifications that were detected were 17p (seven cases; 24\%) and 8q (six cases; 21\%). These observations may indicate early changes during LMS tumorigenesis.

The array CGH analysis results of the current study for a primary tumor indicated that the 8ptel (D8S504 and D8S596) gain and 13q14.2-3 (D13S319 and D13S25) loss were target genes. The loss of 13q14.2-3 has been reported previously (13). The most marked changes (1.25 or 0.75$)$ in the metastatic tumor occurred in 17 DCNAs. Among these loci, WI-5663 (1q21), SHGC-5557 (12ptel), 282M15/SP6 (17ptel) and RUNX1 (21q22.3) were observed in our previously reported case of metastatic osteosarcoma (10).

The current study reports the DCNA observations for a primary tumor and metastatic lymph node in a patient with P-LMS. The gain of 8ptel (D13S319) and the loss of 13q14.2-14.3 (D13S25) may be involved in tumor progression and metastasis. However, further cytogenetic study is required to elucidate the DCNAs in P-LMS.

\section{Acknowledgements}

The authors would like to thank Akimi Sano from the Department of Orthopaedics (University of Toyama, Toyoma, Japan) who provided technical advice. The current study was partly supported by a Grant-in-Aid for Scientific Research from the Japan Society for the Promotion of Science (grant no. 22501043).

\section{References}

1. Sandberg AA and Bridge JA (eds): Leiomyosarcomas. In: The Cytogenetics of Bone and Soft Tissue Tumors. RG Landes Company, Austin, TX, pp104-124, 1994.

2. Sreekantaiah C, Davis JR and Sandberg AA: Chromosomal abnormalities in leiomyosarcomas. Am J Pathol 142: 293-305, 1993.

3. Pinkel D, Segraves R, Sudar D, et al: High resolution analysis of DNA copy number variation using comparative genomic hybridization to microarrays. Nat Genet 20: 207-211, 1998.

4. Pollack JR, Perou CM, Alizadeh AA, et al: Genome-wide analysis of DNA copy-number changes using cDNA microarrays. Nat Genet 23: 41-46, 1999.

5. El-Rifai W, Sarlomo-Rikala M, Knuutila S and Miettinen M: DNA copy number changes in development and progression in leiomyosarcomas of soft tissues. Am J Pathol 153: 985-990, 1998.

6. Fletcher CD: Pleomorphic malignant fibrous histiocytoma: fact or fiction? A critical reappraisal based on 159 tumors diagnosed as pleomorphic sarcoma. Am J Surg Pathol 16: 213-228, 1992.

7. Schürch W, Bégin LR, Seemayer TA, et al: Pleomorphic soft tissue myogenic sarcomas of adulthood: A reappraisal in the mid-1990s. Am J Surg Pathol 20: 131-147, 1996.

8. Yamamoto I, Oshiro Y,Fukuda T and Tsuneyoshi M: Pleomorphic leiomyosarcoma of the soft parts: a reassessment by histology and immunohistochemistry of pleomorphic soft tissue sarcomas. Oncol Rep 6: 533-537, 1999.

9. Oda Y, Miyajima K, Kawaguchi K, et al: Pleomorphic leiomyosarcoma: clinicopathologic and immunohistochemical study with special emphasis on its distinction from ordinary leiomyosarcoma and malignant fibrous histiocytoma. Am J Surg Pathol 25: 1030-1038, 2001.

10. Yasuda T, Kanamori M, Nogami S, et al: Establishment of a new human osteosarcoma cell line, UTOS-1: cytogenetic characterization by array comparative genomic hybridization. J Exp Clin Cancer Res 28: 26, 2009.

11. Kanamori M, San A, Yasuda T, Hori T and Suzuki K: Array-based comparative genomic hybridization for genomic-wide screening of DNA copy number alterations in aggressive bone tumors. J Exp Clin Cancer Res 31: 100, 2012.

12. Riva P, Dalprá L, Gualandri V, et al: 19 p deletion in recurring leiomyosarcoma lesions from the same patient. Cancer Genet Cytogenet 119: 102-108, 2000.

13. Wang R, Titley JC, Lu YJ, Summersgill BM, Bridge JA, Fisher C and Shipley J: Loss of 13q14-q21 and gain of 5p14-pter in the progression of leiomyosarcoma. Mod Pathol 16: 778-785, 2003. 